\title{
Multilayer Network Analysis of Innovation Intermediaries' Activities
}

\author{
Margherita Russo, Annalisa Caloffi, Riccardo Righi, Simone Righi, \\ and Federica Rossi
}

\begin{abstract}
Policymakers wishing to enhance innovation processes in small and medium-sized enterprises increasingly channel their interventions through innovation intermediaries. However, limited empirical research exists regarding the activities and performance of intermediaries, with most contributions taking a qualitative approach and focusing on the role of intermediaries as brokers. In this paper, we analyse the extent to which innovation intermediaries, through their engagement in different activities, support the creation of communities of other agents. We use multilayer network analysis techniques to simultaneously represent the many types of interactions promoted by intermediaries. Furthermore, by originally applying the Infomap algorithm to our multilayer network, we assess the contribution of the agents involved in different activities promoted by intermediaries, and we identify the emerging multilayer communities and the intercohesive agents that span across several communities. Our analysis highlights the potential and the critical features of multilayer analysis for policy design and evaluation.
\end{abstract}

The Authors are members of CAPP-Centro di Analisi delle Politiche Pubbliche, Departiment of Economics, University of Modena and Reggio Emilia, Italy.

M. Russo ( $\square)$

Department of Economics, University of Modena and Reggio Emilia, Modena, Italy

e-mail: margherita.russo@unimore.it
A. Caloffi
Department of Economics and Management, University of Florence, Florence, Italy
R. Righi
European Commission, Joint Research Centre (JRC), Seville, Spain
S. Righi
Department of Computer Science, University College London, London, UK
"Lendület" Research Center for Education and Network Studies (RECENS),
Hungarian Academy of Sciences, Budapest, Hungary
F. Rossi
Birkbeck, University of London, London, UK

(C) Springer Nature Switzerland AG 2020

G. Ragozini, M. P. Vitale (eds.), Challenges in Social Network Research,

Lecture Notes in Social Networks, https://doi.org/10.1007/978-3-030-31463-7_12 
Keywords Multilayer and multiplex networks - Overlapping communities · Regional innovation policy · Innovation intermediaries · Regional innovation system

\section{Issue: Intermediaries in Innovation Processes}

The term 'innovation intermediary' is used in the innovation literature, particularly in the systems-of-innovation perspective, to identify a varied set of organisations whose main mission is to support innovation processes involving other organisations. They are generally needed in the context of rapid changes or when small firms are not able to keep the pace of change in their technical and economic environment. Intermediary functions are performed by private organisations [1], such as innovation consultants [2], innovation brokers [2, 3], and knowledgeintensive business service providers [4], as well as by publicly or public-privately funded organisations such as regional institutions [5], research-industry liaison offices and science parks [6]. Examples of innovation intermediaries funded with substantial public contributions are the Competitiveness Poles in France, the Innovation Networks in Denmark, the Strategic Centres for Science, Technology and Innovation in Finland, the Technology Catapults in the UK, and the Innovation Poles in Italy [7].

Early academic studies of innovation intermediaries emphasised brokering as their central concern. ${ }^{1}$ Subsequent literature has suggested that brokers should be considered 'facilitators of innovation', as they support innovation processes, but the innovations neither originate from nor are transferred by them [8,9]. Other types of intermediaries act as either 'sources of innovation' (i.e. they play a major role in initiating and developing an innovation) or as 'carriers of innovation' (i.e. they transfer an innovation that does not originate from them) [8]. Recent studies move further away from a view of intermediaries as simple providers of connections between other agents positioned at different stages of linear innovation processes, but see intermediaries as enhancing the innovation capacity of many actors embedded within complex systems of interactions. For example, [10] defines innovation intermediaries as 'organisations or groups within organisations that work to enable innovation, either directly by enabling the innovativeness of one or more firms, or indirectly by enhancing the innovative capacity of regions, nations, or sectors'. In the context of their complex role, intermediaries engage in a plurality of activities that can include, among others, technology foresight and technology scouting, supplier selection, research and development (R\&D) partnership formation, technical assistance in the realisation of $\mathrm{R} \& \mathrm{D}$ projects, dissemination and commercialisation of results, and technology transfer. Through all of these activities,

\footnotetext{
${ }^{1}$ For example, Howells [4] proposed a functional definition of innovation intermediary as '[a]n organisation or body that acts [as] an agent or broker in any aspect of the innovation process between two or more parties'.
} 
even those that are not specifically aimed at networking, intermediaries engage with a wide variety of other organisations, and contribute to the formation of communities of organisations that share a common orientation towards open and collaborative innovation. Despite the growing interest in innovation intermediaries, to the best of our knowledge no studies have yet analysed the extent to which intermediaries support the creation of communities of other agents, and the extent to which they do so through the different activities they perform. Thanks to original data relating to a set of publicly funded innovation intermediaries in the region of Tuscany (Italy), we reconstruct the multilayer network of interactions generated by the set of 12 regional innovation intermediaries through a range of different activities that include, among others, the provision of knowledge-intensive services, the setup of collaborative projects, and the promotion of events for members. We then apply the Infomap algorithm [11-13] to detect the emergence of communities around those intermediaries and the actors that play the most central roles within those communities. The composition of communities, by type of agent and by type of activity, and the presence of agents belonging to more than one community are of utmost interest in designing innovation policies that aim to support regional innovation systems. In particular, we explore the following questions:

- Role of agents in information dynamics: to what extent were the innovation intermediaries the most relevant agents in setting up a range of different interactions, and who were, if any, other pivotal agents?

- Structure of communities: What was the structure of the overlapping communities of agents mobilised by the innovation intermediaries?

Our analysis is original with respect to the existing literature, in two main ways. First, we develop an original empirical analysis of intermediaries' activities, using multilayer network representations. Second, we present a new application of the Infomap algorithm for multilayer networks. Such an algorithm allows us to address our two research questions as it provides information regarding (1) the role of each node in the considered network, (2) the detection of communities of agents. Moreover, the use of the Infomap algorithm is particularly appropriate in our context, as information flows are among the elements that determine and influence the formation and the dynamics of socio-economic complex systems, as the one considered in this work [14]. To answer our research questions, this chapter presents the empirical dataset, in Sect. 2, and briefly introduces the multilayer methodology adopted in our analysis, in Sect. 3; Sect. 4 summarises the results with regard to both the centrality of agents in the different networks and the characteristics of emerging communities. Some comments on the Infomap methodology, the policy implications of this approach, and current developments of our research are discussed in Sect. 5. 


\section{Data: Tuscany Policy Programme 2011-2014}

\subsection{The Regional Policy}

Our analysis focuses on a unique database collecting data on innovation intermediaries, funded through a regional policy intervention, in the Italian region of Tuscany. ${ }^{2}$ In the programming period 2007-2013, the regional government of Tuscany funded twelve 'innovation poles'. Each pole was managed by a managing consortium that included several organisations operating in the field of innovation, such as universities, service centres, and enterprises. The managing consortium was led by a leading organisation. In one case only the managing consortium coincided with its leading organisation. Consortium participants would share the use of their research laboratories and facilities and second some of their staff to the poles so that they could carry out specific activities. Poles engaged in the provision of innovation advisory services [15], support to networking or to R\&D activities, and other activities that are typically performed by intermediaries. The poles received public funding to provide these services for free to firms in the region. The regional firms, in order to take advantage of these services, had to become a member of the poles. Poles, which were active in the period July 2011-June 2014, were specialised in different technological areas and applications, as listed in Table 1.

The 3181 members associated to poles were mainly manufacturing enterprises (66.3\%). The remaining share included companies in the service sector: from traditional services (21.9\%) to knowledge-intensive business services (11.8\%). Less than one per cent of the members were other types of organisations (e.g. business

Table 1 The 12 innovation poles: key technologies and applications, consortium participants, and member companies, by type

\begin{tabular}{|c|c|c|c|c|c|c|c|c|c|c|c|c|c|}
\hline \multirow{2}{*}{$\begin{array}{l}\text { id Innovation pole } \\
\text { (consortium's } \\
\text { acronym) }\end{array}$} & \multirow{2}{*}{$\begin{array}{c}\text { Key } \\
\text { technologies } \\
\text { or applications }\end{array}$} & \multicolumn{7}{|c|}{ Organisations managing the consortia } & \multirow{2}{*}{$\begin{array}{c}\text { Members } \\
\text { of the } \\
\text { consortia } \\
\mathrm{N} . \\
\text { (in 2014) }\end{array}$} & \multicolumn{4}{|c|}{$\begin{array}{l}\text { Types of innovation poles' member } \\
\text {-Enterprises }=\text { Service companies } \square \text { KIBS }\end{array}$} \\
\hline & & $\begin{array}{c}\mathrm{N} \\
\text { (including } \\
\text { leader) }\end{array}$ & $\begin{array}{l}\text { Type } \\
\text { University }\end{array}$ & 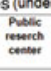 & $\begin{array}{l}\text { Pive: the } \\
\text { Private } \\
\text { reserch } \\
\text { cemert }\end{array}$ & Enterpris & $\begin{array}{l}\text { Toing le } \\
\text { Park } \\
\text { Park }\end{array}$ & $\begin{array}{l}\text { acer) } \\
\text { Senvice } \\
\text { Center }\end{array}$ & & $0 \quad 100200$ & 300400 & 500600 & 700 \\
\hline 12 POLITER & $\begin{array}{l}\text { ICT Technologies, } \\
\text { Telecommunic.\& Robotics }\end{array}$ & 13 & 4 & 6 & & & & $\underline{3}$ & 697 & & & & \\
\hline 7 POLIS & $\begin{array}{l}\text { Technologies for } \\
\text { sustainable cities }\end{array}$ & 8 & 2 & 2 & & & & 4 & 643 & & & $\square$ & \\
\hline 3 OTIR 2020 & $\begin{array}{l}\text { Fashion (textiles, apparel, } \\
\text { leather, shoes, jeweiry) }\end{array}$ & 7 & & & $\underline{2}$ & & & 5 & 501 & & & & \\
\hline 11 POLO12 & $\begin{array}{l}\text { Mechanics, particularly for } \\
\text { automotive and transport }\end{array}$ & 6 & & & 1 & & & $\underline{\mathbf{5}}$ & 390 & & & & \\
\hline 6 PENTA & $\begin{array}{l}\text { Shipbuilding and maritime } \\
\text { technology }\end{array}$ & 5 & & & & 1 & & 4 & 352 & & & & \\
\hline 9 CENTO & $\begin{array}{l}\text { Furniture and interior } \\
\text { design }\end{array}$ & 6 & 1 & & & & & $\underline{5}$ & 322 & & & & \\
\hline 10 PIERRE & $\begin{array}{l}\text { Renewable energies and } \\
\text { energy saving technology }\end{array}$ & 13 & 5 & 2 & 1 & & & $\underline{5}$ & 368 & & & & \\
\hline 2 INNOPAPER & Paper & 1 & & & & & & 1 & 139 & & & & \\
\hline 4 VITA & Life science & 8 & 5 & 1 & & & 1 & 1 & 158 & & & & \\
\hline 8 NANOXM & Nanotechnologies & 6 & 2 & 2 & & & & $\underline{2}$ & 128 & & & & \\
\hline 5 PIETRE & Marble & 4 & 1 & & & 1 & & $\underline{2}$ & 122 & & & & \\
\hline 1 OPTOSCANA & $\begin{array}{l}\text { Optoelectronics for manut. } \\
\text { \& aerospace }\end{array}$ & 2 & & 1 & & & & 1 & 92 & & & & \\
\hline
\end{tabular}

\footnotetext{
${ }^{2}$ The database is available at the following https://doi.org/10.25431/11380_1182469.
} 
associations). The policy was clearly inspired by the Triple Helix approach to innovation, with poles aiming to facilitate the interaction between the three main actors of the innovation system: research, government, enterprises [16]. Moreover, the policymaker was also hoping to promote the development of a regional innovation system [17] that could encourage technology transfer and stimulate the innovation capabilities of regional small and medium-sized enterprises (SMEs). ${ }^{3}$

\subsection{The Interactions Promoted by the Poles}

The innovation poles performed different kinds of activities, which generated interactions among the poles themselves, the poles' leading organisations, and the members of the poles. By monitoring the development of the poles' activities, we have identified the following types of interactions:

- Leadership/management. The interactions between a pole, its managing consortium, and its leading organisation. Note that the same agent (e.g. the same university) can participate in the managing consortia of more than one pole.

- Membership. The interactions between a pole and its members. Note that the same regional actor may become a member of more than one pole, in order to get access to the services provided by the managing consortia of each of them.

- Seconding of staff. The interactions between the staff of the managing consortium (or leading organisation) who are seconded to the pole, as well as those between such workers and their employers.

- Service provision. The interactions between the pole's managing consortium (or leading organisation) and the pole members that benefit from their services.

- Collaboration agreements. The interactions between the poles and the organisations that sign the collaboration agreement.

- Shareholding. The interactions between the pole's managing consortium (or leading organisation) and their shareholders (i.e. those who legally own one or more shares in the organisations managing the consortium).

\subsection{Structuring the Data as a Multilayer Network}

Thanks to the identification of these types of interaction, six layers of a single multilayer network (MLN) are generated. Descriptive statistics for each layer and for the aggregate network are presented in Table 2. Out of the 3986 agents involved overall, we have that: 3305 of them are active in just one layer (for the largest part

\footnotetext{
${ }^{3}$ This information was collected during two interviews (on April 2011 and June 2013) with policymakers who designed and managed the policy.
} 


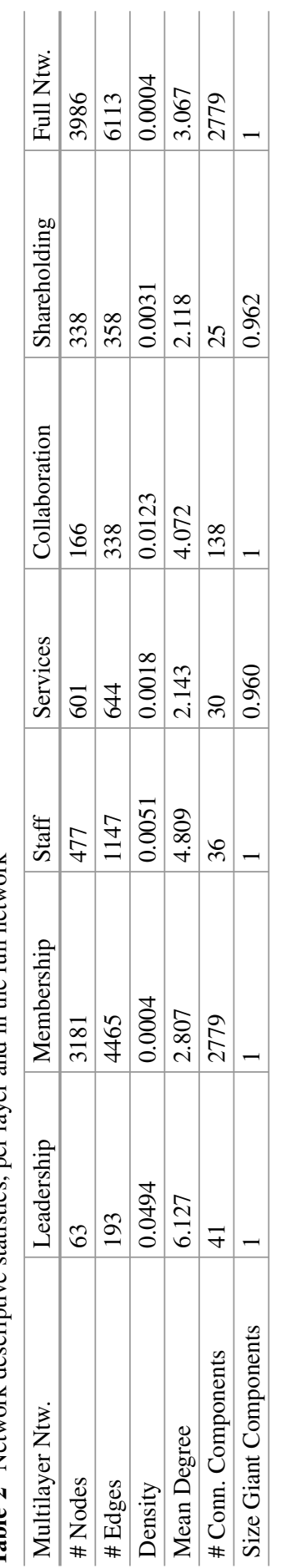



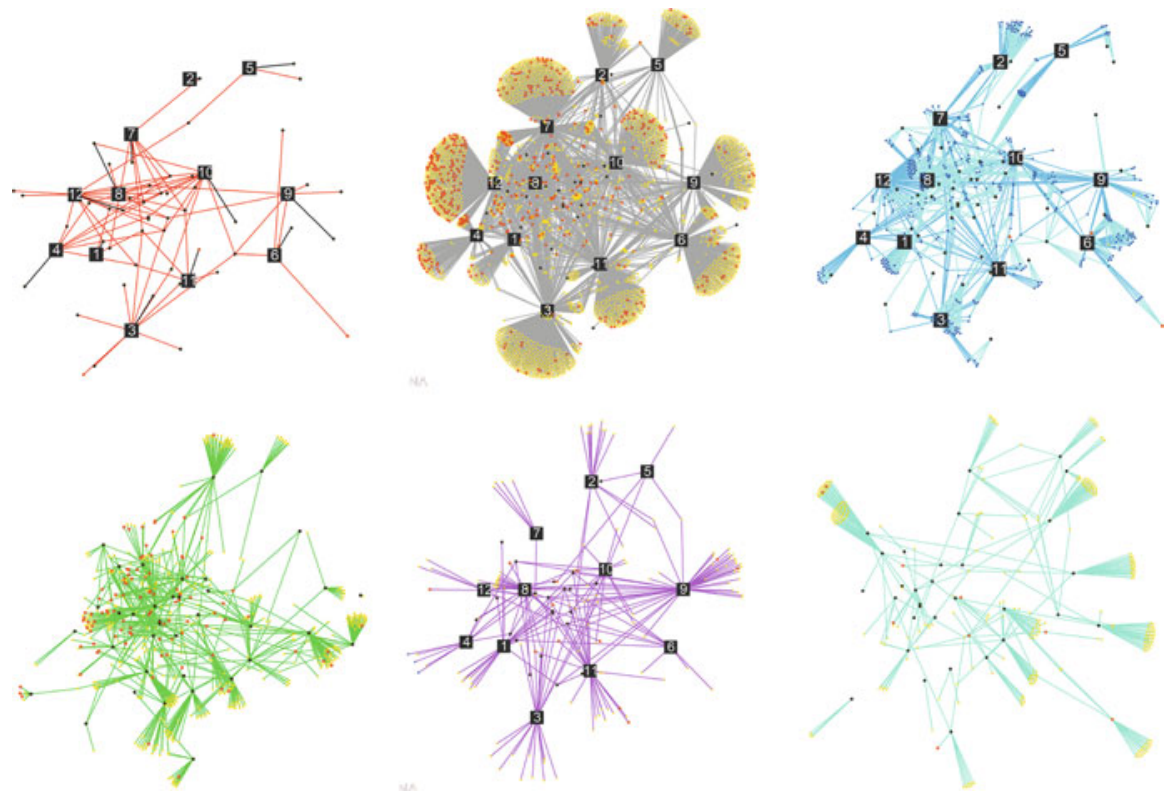

Fig. 1 Graph of 3986 agents, by mode of interaction (layer). Nodes have the same coordinates in all graphs. Nodes' colours: in all graphs, the nodes representing the poles are shown as black squares, with white figures corresponding to 'id' column in Table 1; the nodes representing the leading organisations/managing consortia are black; the nodes representing workers/consultants are blue; the nodes representing KIBS are orange; yellow nodes represent all other types of agents. Top Left Panel: Leading/managing interactions (leading links in black, managing links in red). Top Centre Panel: Membership (linkages in grey). Top Right Panel: Seconding workers (seconding a worker links in light blue, seconded worker links in dark blue). Bottom Left Panel: Service provision (links in green). Bottom Centre Panel: Collaboration agreements (links in pink). Bottom Right Panel: Shareholding (links in aquamarine)

they are members of the poles); 611 of them are active in two layers; 20 of them are active in three layers; 24 of them are active in four layers; 13 of them are active in five layers; 13 of them are active in six layers.

Figure 1 displays the layers. Note that in this visualisation, each node maintains the same coordinates in all the layers in which it is present. Even if the detail of the single nodes is not visible, Fig. 1 gives an overall view from which it is possible to understand that the different layers are characterised by a different relational structure, since each node always maintains the same position in the various layers.

As we are interested in the analysis of the overall activity of intermediaries, we consider all the six layers. However, instead of considering all interactions indistinctly, i.e. just as connections between agents, the MLN allows us to develop an analysis of the distinct dimensions of interactions. 


\section{Methodology}

\subsection{Flows of Information in a Multilayer Network Perspective}

Rosvall and Bergstrom $[11,12]$ introduced a method, based on information theory, to detect communities in complex networks by minimising the length of a twolevel description of a simulated flow circulating through the network. ${ }^{4}$ Such a method is implemented in an algorithm called 'Infomap' [18]. Infomap solves the main problems with Newman and Girvan [19] in identifying communities of very different sizes and, in addition, it allows the detection of overlapping communities $[20,21]$, so that each agent/node can belong to more than one of them. Recently, the Infomap algorithm has been extended by De Domenico et al. [13], so as to run over a multilayer-or multiplex- structure $^{5}$ by

1. considering in each layer just a specific type of relationships (and the agents/ nodes that are involved in these), so as to figure out intra-layer connections;

2. connecting all the projections (i.e. its state-nodes) of the same agent/node (physical-nodes) with inter-layer connections.

Thanks to this implementation, De Domenico et al. [13] show that some features regarding interactions over different dimensions, represented by the layers, can be better investigated and finally unveiled. In addition, as a result of this development, the Infomap multilayer algorithm is set to compute the amount of information flow associated to each state-node, so as to assess the amount of information that agent/nodes collect in each layer in which they have at least one connection. While in network analysis the centrality measures that are most commonly taken into account are betweenness and closeness, in this work we consider the Infomap flow to evaluate agents/nodes centrality in a multilayer structure. As discussed by SoléRibalta and De Domenico [23, p. 76], typical centrality measures, e.g. closeness and betweenness, when implemented in a multilayer network allow for a more correct estimation. ${ }^{6}$ However, only the more sophisticated structure designed by

\footnotetext{
${ }^{4}$ The first level of description concerns the nodes in which the flow moves, and the second level of description concerns sub-areas of the network, i.e. communities, in which the flow tends to circulate for a long period before exiting. Therefore each detected community maximises the probability of the considered random walk to remain within its boundaries before moving into another community.

${ }^{5}$ As reminded by Arenas and De Domenico [22], historically, the term multiplex was coined to indicate the presence of more than one relationship between the same actors of a social network. The terms 'multiplex' and 'multilayer' are used almost indistinctly as they fundamentally refer to the same concept.

${ }^{6}$ Sole-Ribalta and De Domenico [23, p. 76] discuss the problem of overestimation of closeness centrality in an aggregated network, i.e. a network originally formed by several layers that are all aggregated into a single one.
} 
De Domenico et al. [13] makes it possible to disentangle the impact that each layer has in determining the relevance of each agent/node. Based on De Domenico et al.[13], our analysis describes agents/nodes' role by means of the Infomap flow index. Such an index is decomposable not only with regard to the communities to which the agent/node belongs, but especially with regard to the set of layers in which each agent/node is active. Then, we consider the structure of communities generated by nodes' overlaps (i.e. by the presence of agents/nodes belonging to more than one community), which are detected in the MLN, i.e. mesoscale organisations of agents/nodes that can be both bounded in a single layer or that can extend over several layers. In performing such analysis, we investigate: (1) how each node performs in the process of managing information, with respect to the multiple dimensions of interactions in which it is involved (represented by the layers), and (2) the structure of communities emerging through the presence of agents/nodes belonging to more than one of them. Agents that belong to more than one community have been defined as 'intercohesive agents' [24, 25]: these agents are able to create bridges among communities, so they contribute in the sharing of information and competences between them, and they play a determinant role in the evolution of a community structure.

\subsection{Settings of the Algorithm}

We analyse the intermediaries' interactions with a focus on multidimensional links. We model the six types of interactions described above, in Sect. 2 and in Fig. 1, as layers of a MLN. Descriptive statistics of agents' activities in the layers are summarised in Table 2. Agents/nodes are present on a layer if they are involved in at least one interaction of the kind corresponding to the layer. In addition, we also consider inter-layers linkages to connect all the projections (in different layers) of the same agent. All connections (both intra and inter-layers) are undirected and unweighted. The algorithm is asked to identify overlapping communities, thus allowing the nodes to belong to more than one of them. This is necessary for our goals, as it makes it possible to establish the presence of connections among communities (through the overlaps) and, in turn, to investigate the whole structure of communities. Finally, the probability with which the random walk simulated by Infomap can jump, moving from one point of the MLN to another random point of the MLN, is set equal to 0.15 , as in the PageRank algorithm ${ }^{7}$ [26].

\footnotetext{
${ }^{7}$ This is also the value that in $[11,12]$ is used as default value to run and test Infomap.
} 


\section{Main Results}

The multilayer Infomap algorithm allows the assessment of the contribution of each layer and each agent, or groups of agents, to the generation of the total Infomap flow. From Table 3, we observe that the six layers have different importance in generating the information flow: $60 \%$ is generated by the membership to the poles; almost $16 \%$ is due to the network of interactions across poles through workers and consultants; $10.6 \%$ is the share activated by the provision of services from managing organisations to the poles' members; almost $6 \%$ of the Infomap flow reinforces the connections across poles through their indirect links due to the many organisations (mainly local government or public institutions, like Chambers of Commerce) owning shares in the organisations that manage the poles.

Let us now consider the Infomap flow generated by the innovation poles: almost $37 \%$ of the total multilayer flow is generated by the interactions in which poles are engaged, and the largest part of it (almost 30\%) is created through poles' membership. With regard to the 46 organisations leading and managing the poles, we observe that their involvement in the information dynamics, as measured by the Infomap multilayer flow, is due not only to the provision of services to member companies $(5.82 \%)$ and workers to the poles $(4.49 \%)$, but also to the many connections among their shareholders $(3.53 \%)$.

When the multilayer analysis is run, it produces 71 communities, 63 of which are overlapping (Fig.4), with 605 intercohesive agents (15\% of the total) mainly active in two communities. Table 4 lists the types of intercohesive agents, showing that only poles belong to three communities (and only in three cases), while the majority of agents belonging to two communities are manufacturing companies (424 firms) and knowledge-intensive business services (KIBS) (98 firms): manufacturing companies are simply members of two poles, while KIBS are active in demanding services.

The multilayer analysis is informative with regard to the structural aspects of the network: it disentangles communities presenting a higher probability of having

Table 3 Percentage of Infomap flow, by layer and group of agents: poles (first column), leading organisations and 46 organisations belonging to the managing consortia (second column), other agents (third column)

\begin{tabular}{l|c|l|l|c}
\hline \multirow{2}{*}{ Layers } & \multicolumn{2}{l}{ Types of agent } & \multicolumn{2}{l}{} \\
\cline { 2 - 5 } & 12 Poles & Managing consortia & All other organisations & All agents \\
\hline Leadership & 0.93 & 1.83 & 0.04 & 2.8 \\
\hline Membership & 29.66 & 1.28 & 29.30 & 60.2 \\
\hline Staff & 4.16 & 4.49 & 7.21 & 15.9 \\
\hline Services & 0.00 & 5.82 & 4.75 & 10.6 \\
\hline Collaboration & 1.84 & 1.08 & 1.50 & 4.4 \\
\hline Shareholding & 0.00 & 3.53 & 2.57 & 6.1 \\
\hline Total & 36.59 & 18.03 & 45.38 & 100.0 \\
\hline
\end{tabular}


Table 4 Number of nodes, by type, belonging to one, or two, or three communities resulting from the implementation of Infomap over the MLN

\begin{tabular}{l|r|r|l|r}
\hline & \multicolumn{4}{|c}{ \# of communities } \\
\cline { 2 - 5 } & 1 & 2 & 3 & Total \\
\hline Poles & 3 & 6 & 3 & 12 \\
\hline Territorial pubic bodies & 112 & 4 & 0 & 116 \\
\hline Chamber of commerce & 8 & 1 & 0 & 9 \\
\hline University & 78 & 4 & 0 & 82 \\
\hline Public research institutions & 20 & 1 & 0 & 21 \\
\hline Private research institutions & 19 & 0 & 0 & 19 \\
\hline Services centre & 19 & 9 & 0 & 28 \\
\hline Manufacturing company & 1991 & 424 & 0 & 2415 \\
\hline Service company & 194 & 46 & 0 & 240 \\
\hline KIBS & 406 & 98 & 0 & 504 \\
\hline Company association & 67 & 8 & 0 & 75 \\
\hline Other & 44 & 1 & 0 & 45 \\
\hline Workers/consultants & 420 & 0 & 0 & 420 \\
\hline Total & 3381 & 602 & 3 & 3986 \\
\hline
\end{tabular}

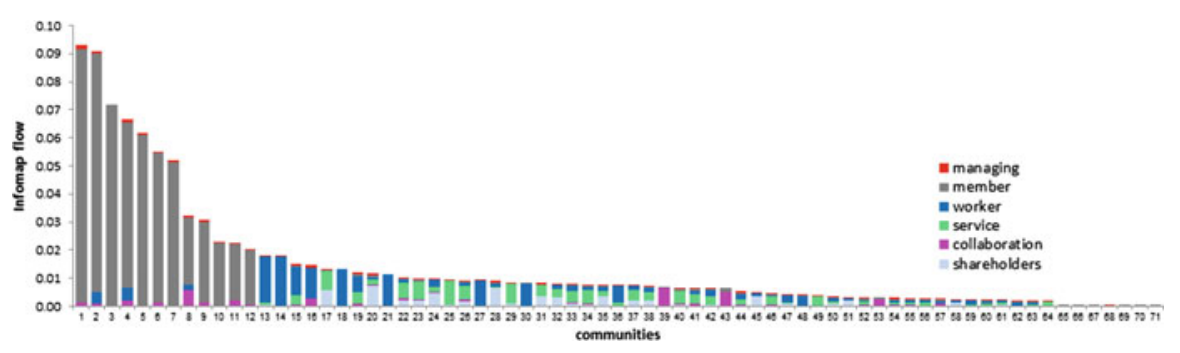

Fig. 2 Multilayer Infomap flow associated to the resulting 71 communities. Colours highlight information regarding the amount of flow generated by each of the six layers. Communities are ordered in decreasing order of total flow

specific connections. Figure 2 shows the relative importance of the multilayer communities and highlights the type of relationships that contributed to their emergence. Figure 3 highlights the composition of communities by type of agent: the first twelve communities (with 3202 agents) account for 62\% of total Infomap flow and have links with the other 60 communities. Each of them has one pole as a pivotal agent. In these communities, the largest share of agents are active only in the layer of membership, the one of the poles they belong to. The analysis shows that most of the agents involved have not exploited the potential of joining the innovation poles. Free membership was not a sufficient incentive to stimulate poles' members demand for services (which was the goal of innovation policy). The organisations that managed the poles and offered the services were only able to exploit part of the demand for services that the poles' members generated. This result, examined in detail by Caloffi et al. [27], opens up the issue of the most appropriate incentives to orient the behaviour of the target beneficiaries of the policy. 


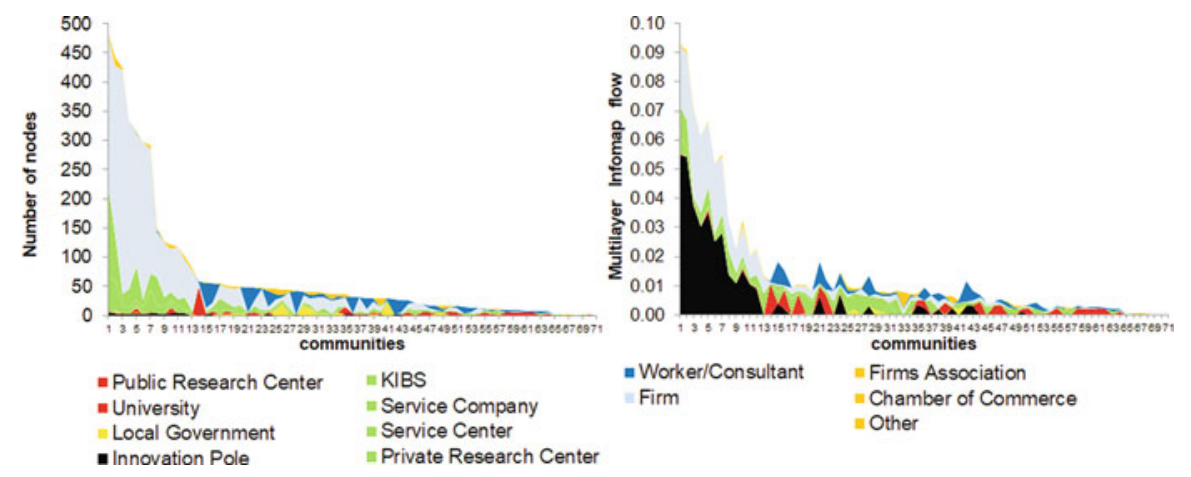

Fig. 3 Left Panel: The 71 multilayer communities: number of agents/nodes by type. Right panel: Multilayer Infomap flow associated to the resulting communities, with information regarding the amount of flow generated by type of agent. Communities are ordered from the largest to the smallest (in terms of number of agents involved)

Overlapping of nodes across communities generates a network structure with 63 communities (out of 71) belonging to the largest component (Fig. 4). The poles largely contribute to the overlapping with other communities, as can be seen from Fig. 4 (where the twelve communities centred on poles are represented only with their label). The other 51 overlapping communities are represented as nodes whose size is proportional to Infomap flow, with each slice representing the share of flow per layer. Edges' width is proportional to the number of agents in common between communities. Results on the overlapping communities provide a way to characterise who are the agents in each community, and the specific activities through which they are connected; a significant result is also the information on which are the most connected communities.

\section{Lessons from the Multilayer Analysis}

Before concluding with a summary of the main results emerging from the Infomap multilayer analysis, some methodological and analytical issues should be stressed with regard to the application of the Infomap multilayer algorithm. First of all, identification of layers is crucial for effective analysis, and weights of linkages are a critical aspect of this analysis. Let us take the case of services: the algorithm computes for this layer $10.6 \%$ of the total multilayer Infomap flow, but when we use all the information in our dataset, distinguishing each type of service provided, we obtain a rather different result. Indeed, Infomap flow is affected by the repetition of an interaction, which is in principle not a problem per se, but we should weight the information flow also for all the other interaction streams under analysis (e.g. by weighting the share of shareholdings, the number of meetings in the board of directors in each consortium, etc.). In our case study, none of these additional 


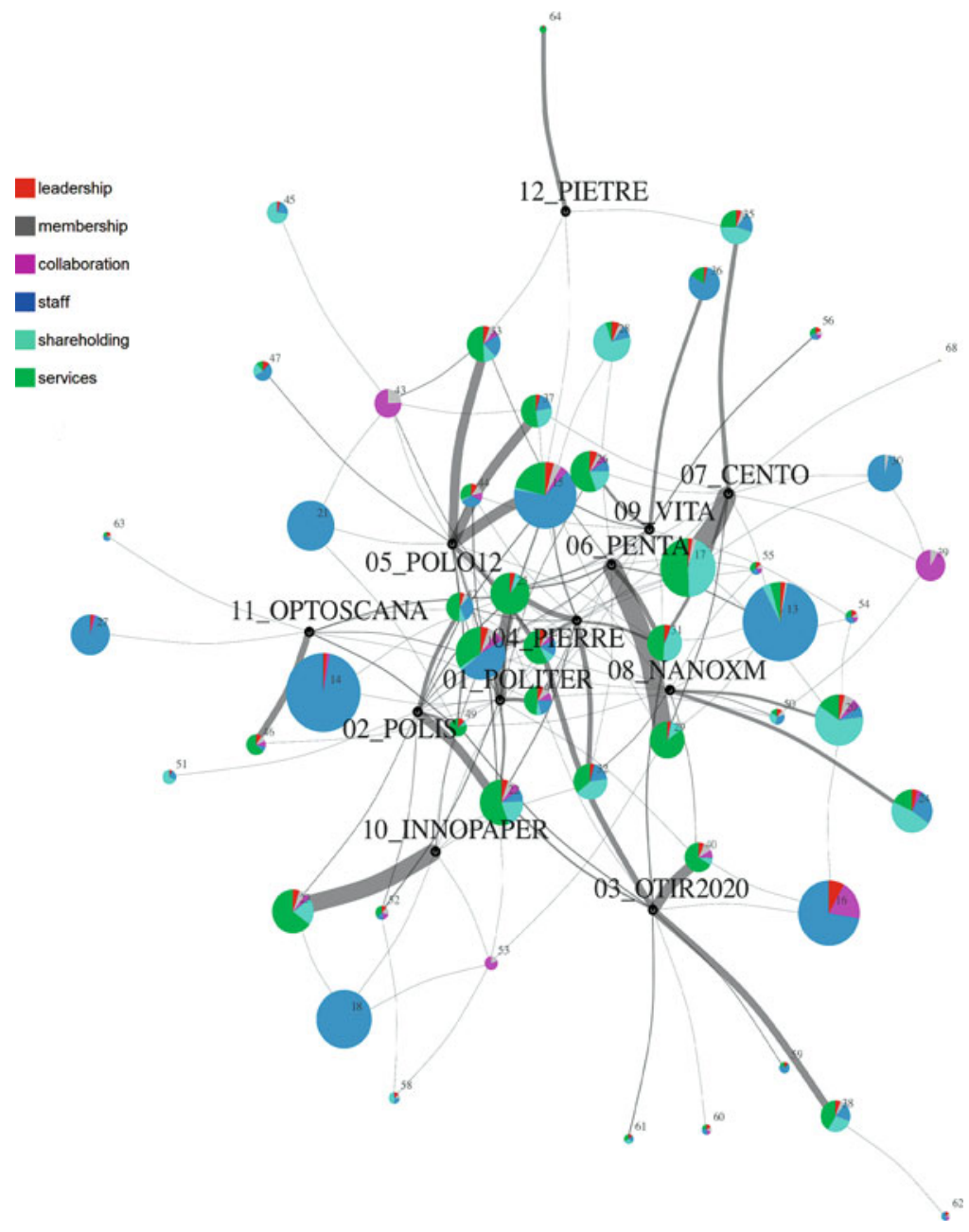

Fig. 4 Network of communities: the largest component encompassing 63 communities. Nodes' size is proportional to Infomap flow. The 12 communities centred on poles' memberships (the largest 12 communities) are not represented in proportion to their size, and they are labelled with the name of the corresponding pole. Slices represent the share of flow by layer (see the legend for the colour by layer). Edges' width is proportional to the number of agents in common between communities

pieces of information would produce a comparable set of weights to be associated to interactions in each layer and this is why we have excluded any reference to weights for all the relationships under analysis. In general, weights of relationships might not be comparable and information might have different granularity. For this 
reason, careful attention must be paid to the identification of layers in implementing the Infomap algorithm. ${ }^{8}$

With regard to our research questions on intermediaries' activities and the design of innovation policy, three main results are drawn by the specific metrics generated by the Infomap multilayer algorithm, which we could not have singled out through any other measure of centrality or method to identify communities of interacting agents. First, the Infomap algorithm allows us to assess to what extent were the innovation poles supported by the multidimensional activity of their managing consortia (universities, research centres, and service centres) and which of these consortia were more effective in mobilising the regional innovation system through the set of specific activities they performed. The organisations participating in the managing consortia generated interactions both within each of the poles in which they were involved (as part of the managing consortium), and across their other connections with other managing consortia or agents embedded in the regional system of innovation (such as KIBS). Moreover, since shareholding was modelled as one of the layers, the Infomap flow weights also the relative importance of the indirect connections enabled by the policy that were provided through the shareholders of the managing consortia involved in the poles.

Second, by distinguishing the amount of information flow generated by the innovation poles through their different activities, we are able to identify which activities contribute most to the generation of information and the creation of communities. We can see, in particular, that simple membership of the poles, rather than access to services, generates most of the information flow. By further disentangling these activities at a more fine-grained level (for example, by distinguishing different types of services), it might be possible to evaluate which of the different poles' activities have a greater impact on the innovation system.

A third result is the detection of overlapping communities of agents mobilised by the regional policy. The Infomap multilayer analysis provides metrics to disentangle in each of the communities the relative importance of the six types of relationship and of the various agents involved. The identification of the resulting intercohesive agents unveils who are the players of the innovation ecosystem that are supported by the regional policy, and through which channels those agents support its potential development. In the design of an innovation policy aiming at enhancing system transformation, the description of all these features might support the definition of more focused criteria to define the activities to implement and the types of agents to involve. The multilayer analysis of the interactions generated by the innovation intermediary could also be usefully deployed as an evaluation instrument in order to illustrate the breadth of engagement of the intermediary and also to compare the different compositions and the different information flows of the MLNs generated by different intermediaries.

\footnotetext{
${ }^{8}$ Directedness, too, may affect the result, although in the case study it did not affect the ranking of agents according to the Infomap flow (results not reported).
} 
Building on these results, a current development of our research is more focused on the economic implications of the emerging structure of communities mobilised by the innovation policy and it concerns the analysis of the economic performance of the intercohesive agents.

Acknowledgements This paper has been developed in the research project 'Poli.in Analysis and modelling of innovation poles in Tuscany' (www.poliinovazione.unimore.it), co-funded by Tuscany's Regional Administration and University of Modena and Reggio Emilia, Italy. For their comments to preliminary versions of this paper, we wish to thank the participants in the 1st EAEPE-RA[X] Workshop 'New Frontiers and Methodological Advances in Cooperation and Network Research', November 2-3, 2015 in Essen, Germany; the Conference 'Networks, Complexity and Economic Development', organised by the Hungarian Academy of Sciences, Research Centre for Economic and Regional Studies MTA KRTK, 30 November to 1 December 2015, Budapest, Hungary; the ARS' 17 Conference, held in Naples, Italy, May 15-17, and the EUSN 2017 Conference, held in Mainz, Germany, September 26-29. Simone Righi acknowledges funding from the European Research Council (ERC) under the European Union's Horizon 2020 research and innovation programme (grant agreement No 648693).

\section{References}

1. Smits, R., Kuhlmann, S.: The rise of systemic instruments in innovation policy. Int. J. Foresight Innov. Policy 1, 4-32 (2004)

2. Klerkx, L., Leeuwis, C.: Establishment and embedding of innovation brokers at different innovation system levels: insights from the Dutch agricultural sector. Technol. Forecast. Soc. Chang. 76, 849-860 (2009)

3. Batterink, M.H., Wubben, E.F.M., Klerkx, L., Omta, S.: Orchestrating innovation networks: the case of innovation brokers in the agri-food sector. Entrep. Reg. Dev. 22(1), 47-76 (2010)

4. Howells, J.: Intermediation and the role of intermediaries in innovation. Res. Policy 35(5), 715-728 (2006)

5. Hargadon, A., Sutton, R.I.: Technology brokering and innovation in a product development firm. Adm. Sci. Q., 42(4), 716-749 (1997)

6. Hansson, F., Husted, K., Vestergaard, J.: Second generation science parks: from structural holes jockeys to social capital catalysts of the knowledge society. Technovation 25(9), 1039-1049 (2005)

7. Russo, M., Caloffi, A., Rossi, F., Fiordelmondo, V., Ghinoi, S.: Evaluating the performance of innovation intermediaries: insights from the experience of Tuscany's innovation poles. ftevalJ. Res. Technol. Policy Eval. 41, 15-24 (2015)

8. Den Hertog, P.: Knowledge-intensive business services as co-producers of innovation. Int. J. Innov. Manag. 4(4), 491-528 (2000)

9. Van Lente, H., Hekkert, M., Smits, R., Van Waveren, B.: Roles of systemic intermediaries in transition processes. Int. J. Innov. Manag. 7(3), 1-33 (2003)

10. Dalziel, M.: Why do innovation intermediaries exist? In: Paper Presented at the 2010 Druid Conference, 16-18 August, Imperial College Business School, London, 2010 (2010)

11. Rosvall, M., Bergstrom, C.T.: An information-theoretic framework for resolving community structure in complex networks. Proc. Natl. Acad. Sci. 104(18), 7327-7331 (2007)

12. Rosvall, M., Bergstrom, C.T.: Maps of random walks on complex networks reveal community structure. Proc. Natl. Acad. Sci. 105(4), 1118-1123 (2008)

13. De Domenico, M., Lancichinetti, A., Arenas A., Rosvall, M.: Identifying modular flows on multilayer networks reveals highly overlapping organization in interconnected systems. Phys. Rev. X, 5(1), 011027 (2015) 
14. Righi, R.: A methodological approach to investigate interactive dynamics in innovative socioeconomic complex systems. Ital. J. Appl. Stat. 30(1), 113-142 (2018)

15. Shapira, P., Youtie, J.: Impact of technology and innovation advisory services. In: Edler, J., Cunningham, P., Gök, A. (eds.) Handbook of Innovation Policy Impact. Edward Elgar Publishing, London (2016)

16. Etzkowitz, H., Leydesdorff, L.: The dynamics of innovation: from National Systems and "Mode 2" to a Triple Helix of university—industry—government relations. Res. Policy 29(2), 109-123 (2000)

17. Cooke, P.N., Heidenreich, M., Braczyk, H.J. (eds.): Regional Innovation Systems: The Role of Governance in a Globalized World. Psychology Press, Colchester (2004)

18. Edler, D., Rosvall, M.: The MapEquation Software Package. http://www.mapequation.org

19. Newman, M.E.J., Girvan, M.: Finding and evaluating community structure in net-works. Phys. Rev. E 69, 26-113 (2004)

20. Fortunato, S., Hric, D.: Community detection in networks: a user guide. Phys. Rep. 659, 1-44 (2016). https://doi.org/10.1016/j.physrep.2016.09.002

21. Kivelä, M., Arenas, A., Barthelemy, M., Gleeson, J.P., Moreno, Y., Porter, M.A.: Multilayer networks. J. Complex Netw. 2, 203-271 (2014). https://doi.org/10.1093/comnet/cnu016

22. Arenas, A., De Domenico, M.: Nonlinear dynamics on interconnected networks. Physica D Nonlinear Phenom. 323-324, 1-4 (2016). https://doi.org/10.1016/j.physd.2016.03.016

23. Solé-Ribalta, A., De Domenico, M., Gómez, S., Arenas, A.: Random walk centrality in interconnected multilayer networks. Physica D Nonlinear Phenom. 324-324, 73-79 (2016). https://doi.org/10.1016/j.physd.2016.01.002

24. Sewell, W.H. Jr.: A theory of structure: duality, agency and transformation. Am. J. Sociol. 98, $1-29(1992)$

25. Vedres, B., Stark, D.: Opening Closure: Intercohesion and Entrepreneurial Dynamics in Business Groups (October 7, 2008) (2008)

26. Brin, S., Page, L.: The anatomy of a large-scale hypertextual web search engine. Comput. Netw. ISDN Syst. 30(1-7), 107-117 (1998)

27. Caloffi, A., Rossi, F., Russo, M.: The emergence of intermediary organizations: a networkbased approach to the design of innovation policies. In: Geyer, R., Cairney, P. (eds.) Handbook on Complexity and Public Policy. Handbooks of Research on Public Policy series, pp. 314331. Edward Elgar Publishing, Cheltenham (2015) 